\title{
Prevention and management of urosepsis triggered by ureteroscopy
}

This article was published in the following Dove Press journal: Research and Reports in Urology

\section{Kymora B Scotland Dirk Lange}

Department of Urologic Sciences, University of British Columbia,

Vancouver, BC, Canada
Correspondence: Dirk Lange Department of Urologic Sciences, University of British Columbia, Vancouver, BC, Canada Email dirk.lange@ubc.ca

\begin{abstract}
Urosepsis is a potentially life-threatening phenomenon that may occur after an ureteroscopic procedure. With the increasingly widespread adoption of ureteroscopy, there is a concern that the rates of urosepsis may increase. This review examines the current work being undertaken to minimize postprocedure infections both in the field of device development and in clinical care. Guidelines for the diagnosis and management of urosepsis will be discussed.

Keywords: urosepsis, sepsis, urinary tract infection, ureteroscopy, ureteroscope, ureteral stent

\section{Introduction}

Urosepsis is a systemic response to and potentially life-threatening sequela of urogenital tract infection. It often results secondary to urinary tract obstruction associated with phenomena such as urolithiasis, tumor and stenosis, but it may also occur after urinary tract manipulation such as ureteroscopy. The development of bacteremia resulting from the dissemination of uropathogenic bacteria into the bloodstream marks the movement of the infection from a localized insult to systemic disease.
\end{abstract}

\section{Defining urosepsis}

The systemic inflammatory response syndrome (SIRS) is a collection of symptoms that have been used to identify patients at high risk for rapid development of sepsis. These symptoms include fever, tachycardia, tachypnea, and elevated white blood cell count. ${ }^{1}$

In recent years, the diagnosis of sepsis has been less dependent on these SIRS criteria. However, they continue to be useful warning signs. There is currently no specific definition of sepsis. This has led to consensus definitions incorporating various laboratory and physiologic parameters. ${ }^{2,3}$ The Third International Sepsis Consensus Definition Task Force defines sepsis as "life threatening organ dysfunction due to dysregulated host response to infection". " Sepsis has also been classified based on the level of severity with patient outcomes worsening as they develop associated organ dysfunction and shock. Patients with refractory septic shock have mortality rates $\sim 50 \%{ }^{5}$

\section{Causes of urosepsis}

Urinary tract infections (UTIs) result from the activity of pathogenic microbes within the urinary system and may be secondary to chronic or acute colonization of the system. The most common uropathogen is Escherichia coli, which is responsible for $\sim 80 \%$ of uncomplicated UTIs or pyelonephritis. ${ }^{6}$ Complicated UTIs such as those resulting 
after ureteroscopy are caused by a more diverse range of microbes encompassing E. coli, Proteus, and Pseudomonas species, Serratia and group B Streptococci, as well as nonbacterial pathogens including various Candida species. There is also the worsening spread of multidrug-resistant infections, particularly in patients with recurrent UTI and treatment histories. ${ }^{7}$ UTIs account for $40 \%$ of the cases of sepsis secondary to nosocomial infections. ${ }^{8}$

Urosepsis typically begins with a UTI and most often follows pyelonephritis. ${ }^{9}$ It may also result from infection originating in the male genital tract, particularly the prostate. The severity of urosepsis will be primarily dependent upon the host response. Patients with risk factors for UTI formation include immunocompromised patients such as post-transplantation, diabetic, or otherwise immunosuppressed individuals. Elderly patients are also vulnerable. Patients who are particularly susceptible to infection include those with anatomic abnormalities within the collecting system, chronic drains including Foley catheters, nephrostomy tubes and ureteral stents as well as patients with a history of infectious calculi or prior urologic interventions. ${ }^{9}$

\section{Cost of urosepsis}

The patient and economic costs of sepsis are substantial. Mortality rates range from $20 \%$ to $50 \%$ with septic shock being the major cause of patient demise in intensive care units as well as the primary reason for admission to these units..$^{9,10}$ Sepsis is an expensive health care issue with costs $>\$ 24$ billion per year in the USA. ${ }^{11}$ A more recent statistical brief from the Healthcare Cost and Utilization Project revealed that sepsis accounted for $6.2 \%$ of all hospital costs. ${ }^{12}$ Urosepsis accounts for $25 \%$ of all adult septic episodes. It can lead to septic shock in a minority of cases. ${ }^{13}$ While there is a dearth of data on the rate of ureteroscopy-triggered urosepsis to date, a previous retrospective study of patients treated for uroseptic shock revealed that urosepsis followed urologic interventions in $17 \%$ of patients. ${ }^{14}$ The risk of infectious complications following ureteroscopy has been documented and ranges from $2.2 \%$ to $20 \%$ in several studies.${ }^{15-18}$ Although mortality following ureteroscopy is rare, a recent multi-institutional case report of six postureteroscopy mortalities revealed that four of those patients died as a result of urosepsis. ${ }^{19}$ This underscores the seriousness of an episode of urosepsis when it occurs. The concerted effort required for the diagnosis and effective management of urosepsis accounts for the significant economic cost.

\section{Clinical presentation}

Urosepsis is a clinical syndrome. It can present with various symptoms including fever and tachypnea as well as acute multiorgan dysfunction and hypotension requiring pharmacologic support (Table 1). It is important to note that urosepsis can progress rapidly in severity and must be aggressively managed. ${ }^{9}$

The initiating insult for urosepsis is a local infection of the genitourinary tract. Sepsis results from an uncontrolled systemic response to that infection. The early clinical presentation may involve temperature extremes (fever or hypothermia), tachycardia, or altered mental status. Patients may develop respiratory alkalosis secondary to tachypnea. Those with worsening symptoms often demonstrate oliguria, hypotension, laboratory abnormalities including elevated white blood cells, and coagulation dysfunction. If not controlled, patients may develop multiorgan dysfunction, which can eventually prove fatal.

\section{Pathogenesis}

The triggering event for urosepsis has been postulated to be the prompting of the host inflammatory response by the presence of intact bacteria or bacterial cell wall

Table I Clinical presentation of urosepsis

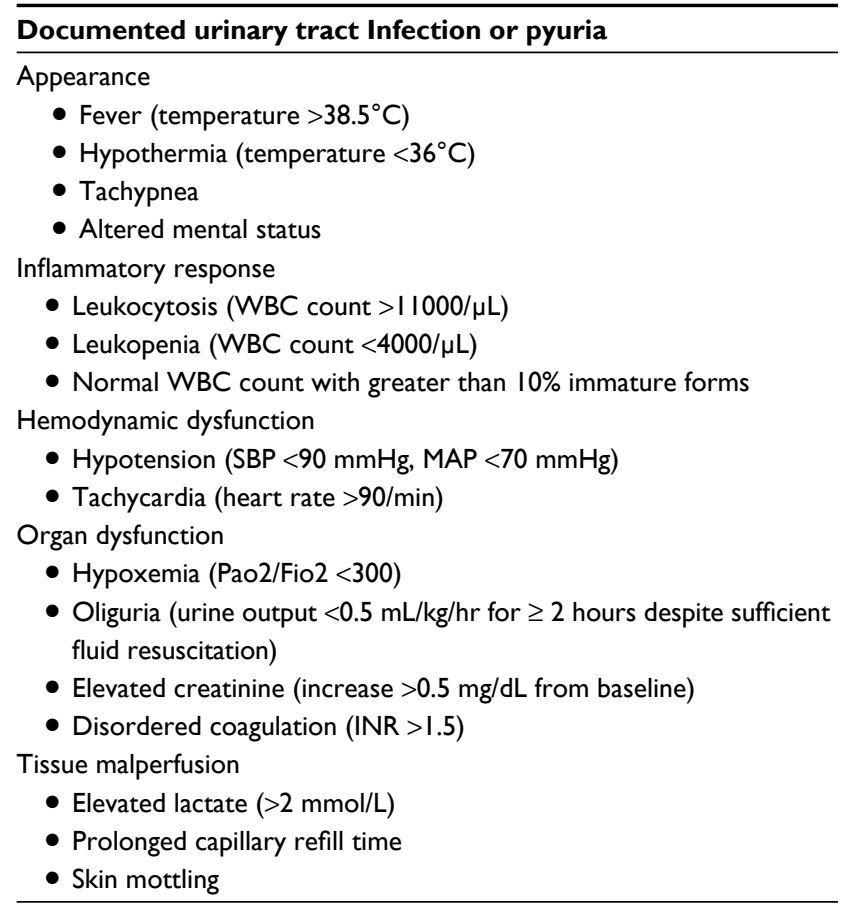
Note: Data from Wagenlehner et al..$^{13}$

Abbreviations: MAP, mean arterial pressure; WBC, white blood cells; INR, international normalized ratio; aPTT, activated partial thromboplastin time; SBP, systolic blood pressure. 
components within the urogenital tract. ${ }^{14}$ They may be present secondary to manipulation during ureteroscopy but have also been thought to be associated with the presence of ureteral stents placed at the conclusion of the procedure. The formation of biofilm on urinary drainage devices such as ureteral stents and Foley catheters is thought to play a role in the genesis of urosepsis. Biofilm is a thin layer formed by the accumulation of microbes and bacterial cell wall portions as well as extracellular biopolymers establishing a matrix..$^{20}$ The first step of biofilm formation is the development of the conditioning film. ${ }^{21}$ This layer coats foreign bodies placed in the body including ureteral stents. Biofilm formation is thought to exacerbate urothelial irritation, leading to an inflammatory response. ${ }^{21}$ The development of biofilm has also been postulated to attract further bacterial adhesion, possibly leading to infection ${ }^{22}$ or predisposing patients undergoing subsequent ureteroscopic intervention to infection. ${ }^{23}$ However, we and others have shown no statistically significant association between biofilm formation and increased bacterial adhesion in vitro ${ }^{24}$ or postoperative infection in patients..$^{25}$ These data suggest that the presence of biofilm may not necessarily result in the development of an UTI, particularly in those patients with intact immune systems.

The colonization of ureteral stents by bacteria occurs in $42 \%-90 \%$ of patients. ${ }^{23}$ Bacteria will adhere to stents in both the presence and absence of conditioning film. ${ }^{24}$ The subsequent development of UTIs was shown to be dependent on stent dwell time. ${ }^{26}$ However, stent colonization tends to be polymicrobial while UTIs generally result from a single strain of bacteria, implying that stent-associated infection is likely not a straightforward process, potentially resulting from contamination during the insertion. ${ }^{27}$

We have championed the use of coated biodegradable stents as a mean of addressing the issue of stent-associated UTIs. One benefit of stent degradation would be the ease of disposal via the urine. The concept of the drug-eluting or coated stent holds that altering the surface of the stent would modify its resistance to bacterial adhesion, therefore decreasing the chance of infection. ${ }^{28}$ Work is ongoing to develop suitable coated stents using biomaterials, which would combat infection without inducing antibiotic resistance.

There is a paucity of systematic reviews of ureteroscopytriggered urosepsis. A recent prospective study by Blackmur et al demonstrated a statistically significant association between urosepsis and a history of diabetes, ischemic heart disease, American Society of Anesthesia (ASA) score, and stone volume on univariable analysis. The study found that patients at highest risk for urosepsis status post ureteroscopy are those with preoperative positive urine samples. This correlation maintained significance on multivariable analysis. ${ }^{16}$ The association held irrespective of preoperative antibiotic treatment or whether the patient was asymptomatic. This result was corroborated by another group who found an association between preoperative pyuria and postoperative febrile UTIs. ${ }^{17}$ Recent work from the Clinical Research Group of the Endourological Society (CROES) study also provided data demonstrating that cardiovascular disease and ASA score were significantly associated with postoperative UTI. This prospective multi-institutional study also showed that preoperative prophylactic antibiotics in patients with a negative baseline urine culture do not reduce postoperative febrile UTIs. ${ }^{18}$ Given these data, potential risk factors for this phenomenon of urosepsis following ureteroscopy are a positive preoperative urine culture, stone burden, and comorbid conditions. However, there are several inconsistencies in the findings of various similar studies to date. Blackmur found an association between same session bilateral ureteroscopy and urosepsis, a finding which is in direct contrast to work presented by Hollenbeck et al. ${ }^{29}$ Similarly, Mitsuzuka et al ${ }^{17}$ proposed that preoperative acute pyelonephritis played a role in postoperative febrile UTI, while the opposite was found by Blackmur. This lack of consensus in results of the studies done may be due to the fact that they are primarily small or single-institution reports. A prospective multi-institutional study would help to clarify this issue.

The pathogenesis of urosepsis is not simply that of a worsening UTI. It is indeed complex with a series of interactions beginning with an initial inflammatory response and a subsequent anti-inflammatory counter regulatory response resulting in an eventual overwhelming immunosuppression. ${ }^{13}$ The overall patient response incorporates the autonomic nervous system, coagulatory pathway, and endocrine system. The complex interregulatory processes involved in urosepsis continue to be elucidated, and a discussion of the various pathways is beyond the scope of this review.

\section{Management Diagnosis of urosepsis}

As mentioned earlier, there is no specific definition of urosepsis. The Sepsis Consensus Definition Task Force introduced the sequential (sepsis-related) organ failure assessment (SOFA) as a mean of operationalizing this most recent consensus definition of sepsis and provided the quick SOFA (qSOFA) scoring system, which facilitates the bedside diagnosis of sepsis. ${ }^{4}$ However, early attempts at the validation 
of this model have produced mixed results. ${ }^{30,31}$ Diagnosis continues to rely on recognition of the constellation of symptoms associated with sepsis (Table 1). In addition to the discussion of subjective fevers, an adequate history must query symptoms of suprapubic or flank discomfort, dysuria, and irritative voiding.

\section{Treatment of urosepsis}

The most commonly advocated treatment plan for urosepsis is the protocol for early recognition and immediate supportive and counter-infection activity advocated by the Surviving Sepsis Campaign. ${ }^{32}$ Critical to the management of urosepsis is the timeframe within which the initial diagnosis is reached and treatment measures are undertaken. Patients presenting with concerning symptoms status post ureteroscopy should have microbiological sampling in the form of urine and blood cultures obtained immediately and supportive measures initiated. Serum procalcitonin may also be obtained at this point. Procalcitonin is a marker of inflammation, particularly in response to a stimulus of bacterial origin. ${ }^{30}$ It is an acute phase reactant that is often elevated during a systemic response to infection as occurs in sepsis. Thus, it has been used as an early marker of severe bacterial infection ${ }^{33}$ and was recently shown to have some utility as an indicator of urosepsis. ${ }^{34,35}$ Because rapid diagnosis of urosepsis is essential for effective therapy, procalcitonin levels may prove a useful tool for focusing the differential diagnosis.

Increased rates of mortality are associated with delayed initiation of antimicrobials. ${ }^{36}$ Hence, patients with a presentation concerning for urosepsis should have early institution of antibiotics. On initial patient presentation, there may be an absence in previous pathogen speciation. Once culture specimens are obtained, empiric treatment with suitably broad-spectrum antibiotics should be started in the concerning patient. ${ }^{13}$ This is done in an effort to combat as many likely pathogens as possible. ${ }^{37}$ Once the offending microbes are known, more specific antibiotics can be given. Antibiotic choice should also be guided by preoperative antibiotic history and perioperative details as well as local and institutional microbial susceptibility patterns. It is imperative that the local antibiogram is consulted so that bacterial resistance patterns are taken into consideration and ineffective antibiotics are avoided. ${ }^{38}$ The use of appropriately dosed and chosen antibiotics has been shown to improve outcomes in patients with urosepsis and septic shock. ${ }^{39}$ Particularly in the case of multidrug-resistant organisms, the choice of appropriate antibiosis will depend on an understanding of common mechanisms of resistance of the most likely pathogens. ${ }^{37}$ Organisms are rapidly developing resistance to even broad-spectrum antibiotics, resulting in a weakened armamentarium. Management of these infections will often require a multidisciplinary approach involving infectious disease specialists.

Management will also include aggressive resuscitation and supportive care with close monitoring. This will likely require a multidisciplinary approach. The Surviving Sepsis Campaign organized the management of sepsis into treatment bundles each with a timeframe for implementation. ${ }^{32}$ Hence, the treatment of urosepsis can be undertaken in a systematic fashion with special emphasis on early goal-directed therapy. ${ }^{40}$ This treatment paradigm advocates for the fluid resuscitation of patients who are hypoperfused or present with elevated serum lactic acid levels via a protocol aimed at maintaining several parameters within specific limits. ${ }^{13}$ The key recommendations are presented in Figure 1.

A thorough history should reveal previous episodes of UTI with urine culture results and preprocedure antibiotic use. A timeline of symptoms should be established for prompt identification and treatment of sepsis. Patients must then be monitored closely for the development of organ dysfunction.

Before attributing sepsis to a recently performed ureteroscopy, a thorough physical examination must be undertaken

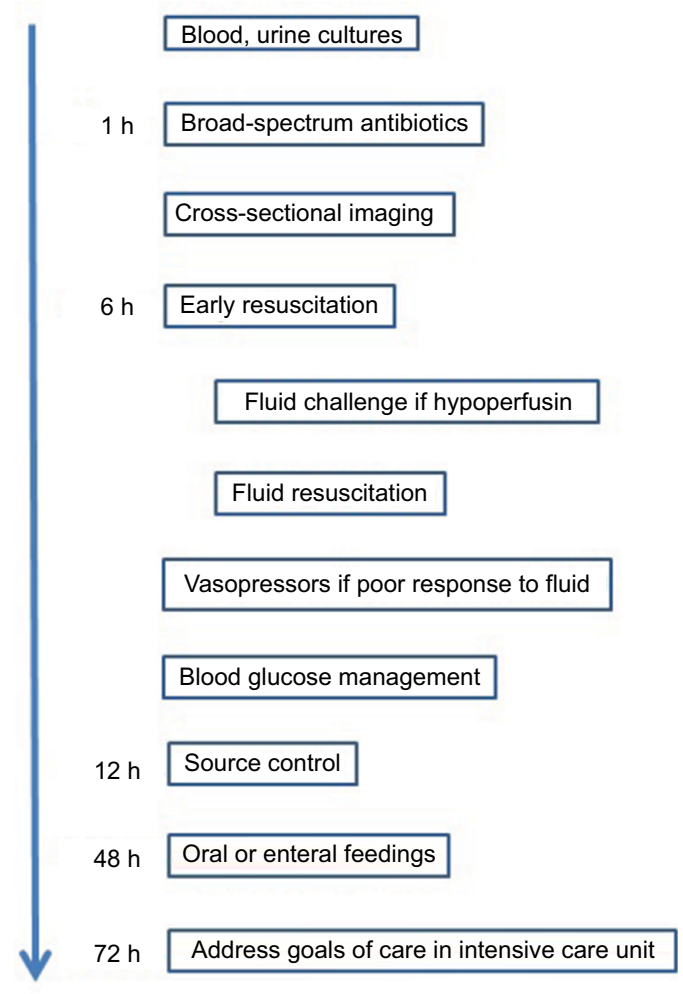

Figure I Clinical management of urosepsis. 
including a digital rectal examination in males to rule out acute prostatitis and palpation of the testes to rule out epididymoorchitis. All patients should undergo palpation of suprapubic and flank regions. Patients should undergo cross-sectional imaging in order to query the presence of urinary tract injury or postprocedural collections as a source of infection after ureteroscopy. For urosepsis in particular, the ability to decompress the urinary system with the use of stents, percutaneous nephostomy tubes, or urinary catheters in the event of obstructive uropathy is critical. ${ }^{9}$

The issue of source control must also be considered. The development of urosepsis following ureteroscopy may occur in patients with ureteral stents placed perioperatively. ${ }^{28}$ Discussions should be undertaken with respect to the timely removal or exchange of foreign bodies such as stents and Foley catheters. Patients with chronic urinary system drainage as provided by ureteral stents and bladder catheters are predisposed to bacteriuria and funguria. The subset of patients with chronic funguria may be susceptible to the development of urosepsis status postureteroscopy. This funguria will be more often seen in patients with some level of immune system compromise such as diabetics or patients who have received extended antimicrobial therapy. Patients with urosepsis secondary to fungemia require antifungal treatment in the form of azoles or systemic amphotericin B in azole-refractory infection. ${ }^{41}$

\section{Prevention}

Urosepsis prevention involves identifying comorbidities or genitourinary abnormalities that predispose a patient to infection. Patients with diabetes and other diseases or treatment regimens causing immunosuppression must be closely monitored in the intraoperative and early postoperative periods. Congenital malformations such as ureteropelvic junction obstruction or presentations such as neurogenic bladder with often concomitant bladder dysfunction and vesicoureteral reflux are also associated with an increased risk of UTIs. ${ }^{42,43}$ Appropriate perioperative antimicrobials should be used, and postureteroscopy Foley catheters should be placed with caution and kept only as long as they are necessary for urinary tract drainage.

Ureteroscopy and other procedures that involve instrumentation of the genitourinary tract have an elevated risk of postoperative UTI development. ${ }^{44}$ This risk is even more elevated in patients with positive preoperative urine cultures, the presence of foreign bodies within the urinary tract, obstruction, history of urinary diversion and comorbidities such as diabetes and paraplegia. ${ }^{25}$ Periprocedural antibiotics have been shown to decrease UTI in patients undergoing ureteroscopy ${ }^{44}$ and have since been recommended by American Urological Association practice statements. ${ }^{45} \mathrm{We}$ have recently demonstrated that a single dose of antibiotic is generally sufficient to achieve this..$^{25}$ These data suggest that preoperative urine cultures are imperative and that positive cultures must be adequately treated. Furthermore, patients with positive preoperative cultures should be more closely monitored postoperatively.

Intraoperative stone cultures have also proven useful in identifying the microbial source of urosepsis in patients who have undergone lithotripsy for endoscopic stone treatment. ${ }^{46}$ A retrospective study by Eswara et $\mathrm{al}^{46}$ revealed that $73 \%$ of patients who developed sepsis after ureterscopy or percutaneous nephrolithotomy had positive stone cultures in the absence of positive preoperative urine cultures. This supports the notion that stone cultures should be routinely sent in ureteroscopy patients.

There has been increasing concern over the possibility of infections due to ureteroscopes themselves. In the case of reusable endoscopes, this may be due to problems with reprocessing as well as ureteroscope defects. ${ }^{47,48}$ A recent prospective study demonstrated multiple instances of contamination even when institutional reprocessing protocols were designed to be consistent with guidelines. ${ }^{49}$ Several incidents of deviation from guideline recommendations were noted. Safe decontamination of reusable ureteroscopes will require mandatory adherence to guidelines for reprocessing. ${ }^{50}$ In addition, protocols should be instituted that include repeated evaluations of reprocessing practices and routine examination of ureteroscopes for infectious material. Concerns for persistent contamination even in institutions with strict adherence to guidelines have led to the championing of disposable uereteroscopes. ${ }^{51}$ Infection prevention may be improved with the adoption of these sterile single use devices.

Several intraoperative practices such as the use of ureteral access sheaths and low pressure irrigation devices have been proposed anecdotally to decrease postoperative complications. However, to our knowledge, there are few peer-reviewed English language studies supporting these practices. ${ }^{52}$ In fact, a systematic review of ureteral access sheaths reports an association with increased risk of postoperative complications. ${ }^{53}$ The concern for renal pelvic pressures stems from the notion that persistent pressures in excess of normal range may be attained during ureteroscopy and that this may increase the risk of postoperative infection. Although there are currently no systematic studies supporting these practices, we propose that it is good clinical practice 
to limit operative time and to endeavor to maintain low irrigation pressures as well as consistent decompression of the upper urinary tract.

\section{Conclusion}

Ureteroscopy-associated urosepsis is a nosocomial infection. With the increasing popularity of ureteroscopic procedures, it is imperative that patients are administered appropriate perioperative antimicrobial prophylaxis in order to decrease the risk of postprocedure UTI and urosepsis. The development of urosepsis after ureteroscopy is a potentially life-threatening phenomenon, and suspected sepsis must be speedily and aggressively managed.

\section{Disclosure}

The authors report no conflicts of interest in this work.

\section{References}

1. Bone RC, Balk RA, Cerra FB, et al. Definitions for sepsis and organ failure and guidelines for the use of innovative therapies in sepsis. The ACCP/SCCM Consensus Conference Committee. American College of Chest Physicians/Society of Critical Care Medicine. Chest. 1992;101(6):1644-1655.

2. Rhodes A, Evans LE, Alhazzani W, et al. Surviving Sepsis Campaign: international guidelines for management of sepsis and septic shock: 2016. Intensive Care Med. 2017;43(3):304-377.

3. Singer M. The new sepsis consensus definitions (Sepsis-3): the good, the not-so-bad, and the actually-quite-pretty. Intensive Care Med. 2016;42(12):2027-2029.

4. Singer M, Deutschman CS, Seymour CW, et al. The Third International Consensus Definitions for sepsis and septic shock (Sepsis-3). JAMA. 2016;315(8):801-810.

5. Caironi P, Tognoni G, Masson S, et al. Albumin replacement in patients with severe sepsis or septic shock. N Engl J Med. 2014;370(15):1412-1421.

6. Dielubanza EJ, Schaeffer AJ. Urinary tract infections in women. Med Clin North Am. 2011;95(1):27-41.

7. Barber AE, Norton JP, Wiles TJ, Mulvey MA. Strengths and limitations of model systems for the study of urinary tract infections and related pathologies. Microbiol Mol Biol Rev. 2016;80(2):351-367.

8. Johansen TE, Cek M, Naber KG, Stratchounski L, Svendsen MV, Tenke P. Prevalence of hospital acquired urinary tract infections in urology departments. Eur Urol. 2007;51(4):1100-1112.

9. Wagenlehner FM, Pilatz A, Weidner W, Naber KG. Urosepsis: overview of the diagnostic and treatment challenges. Microbiol Spectr. 2015;3(5).

10. Angus DC, Linde-Zwirble WT, Lidicker J, Clermont G, Carcillo J, Pinsky MR. Epidemiology of severe sepsis in the United States: analysis of incidence, outcome, and associated costs of care. Crit Care Med. 2001;29(7):1303-1310.

11. Lagu T, Rothberg MB, Shieh MS, Pekow PS, Steingrub JS, Lindenauer PK. Hospitalizations, costs, and outcomes of severe sepsis in the United States 2003 to 2007. Crit Care Med. 2012;40(3):754-761.

12. Torio C (AHRQ), Moore B (Truven Health Analytics). National Inpatient Hospital Costs: The Most Expensive Conditions by Payer, 2013. HCUP Statistical Brief \#204. Rockville, MD: Agency for Healthcare Research and Quality; 2016. Available from: http://www.hcup-us.ahrq. gov/reports/statbriefs/sb204-Most-Expensive-Hospital-Conditions.pdf. Accessed May 4, 2018.

13. Wagenlehner FM, Lichtenstern C, Rolfes C, et al. Diagnosis and management for urosepsis. Int J Urol. 2013;20(10):963-970.

14. Wagenlehner FM, Pilatz A, Naber KG, Weidner W. Therapeutic challenges of urosepsis. Eur J Clin Invest. 2008;38(suppl 2):45-49.
15. Sohn DW, Kim SW, Hong CG, Yoon BI, Ha US, Cho YH. Risk factors of infectious complication after ureteroscopic procedures of the upper urinary tract. J Infect Chemother. 2013;19(6):1102-1108.

16. Blackmur JP, Maitra NU, Marri RR, Housami F, Malki M, McIlhenny C. Analysis of factors' association with risk of postoperative urosepsis in patients undergoing ureteroscopy for treatment of stone disease. $J$ Endourol. 2016;30(9):963-969.

17. Mitsuzuka K, Nakano O, Takahashi N, Satoh M. Identification of factors associated with postoperative febrile urinary tract infection after ureteroscopy for urinary stones. Urolithiasis. 2016;44(3):257-262.

18. Martov A, Gravas S, Etemadian M, et al; Clinical Research Office of the Endourological Society Ureteroscopy Study Group. Postoperative infection rates in patients with a negative baseline urine culture undergoing ureteroscopic stone removal: a matched case-control analysis on antibiotic prophylaxis from the CROES URS global study. J Endourol. 2015;29(2):171-180.

19. Cindolo L, Castellan P, Scoffone CM, et al. Mortality and flexible ureteroscopy: analysis of six cases. World J Urol. 2016;34(3): 305-310.

20. Goto T, Nakame Y, Nishida M, Ohi Y. Bacterial biofilms and catheters in experimental urinary tract infection. Int $J$ Antimicrob Agents. 1999;11(3-4):227-231.

21. Zumstein V, Betschart P, Albrich WC, et al. Biofilm formation on ureteral stents - incidence, clinical impact, and prevention. Swiss Med Wkly. 2017;147:w14408.

22. Choong $\mathrm{S}$, Whitfield $\mathrm{H}$. Biofilms and their role in infections in urology. BJU Int. 2000;86(8):935-941.

23. Kehinde EO, Rotimi VO, Al-Hunayan A, Abdul-Halim H, Boland F, Al-Awadi KA. Bacteriology of urinary tract infection associated with indwelling J ureteral stents. J Endourol. 2004;18(9):891-896.

24. Elwood CN, Lo J, Chou E, et al. Understanding urinary conditioning film components on ureteral stents: profiling protein components and evaluating their role in bacterial colonization. Biofouling. 2013;29(9):1115-1122.

25. Chew BH, Flannigan R, Kurtz M, et al. A single dose of intraoperative antibiotics is sufficient to prevent urinary tract infection during ureteroscopy. J Endourol. 2016;30(1):63-68.

26. Kehinde EO, Rotimi VO, Al-Awadi KA, et al. Factors predisposing to urinary tract infection after $\mathrm{J}$ ureteral stent insertion. $\mathrm{J}$ Urol. 2002;167(3):1334-1337.

27. Lange D, Bidnur S, Hoag N, Chew BH. Ureteral stent-associated complications - where we are and where we are going. Nat Rev Urol. 2015;12(1): $17-25$.

28. Lo J, Lange D, Chew BH. Ureteral stents and foley catheters-associated urinary tract infections: the role of coatings and materials in infection prevention. Antibiotics (Basel). 2014;3(1):87-97.

29. Hollenbeck BK, Schuster TG, Faerber GJ, Wolf JS Jr. Safety and efficacy of same-session bilateral ureteroscopy. J Endourol. 2003;17(10):881-885.

30. Askim Å, Moser F, Gustad LT, et al. Poor performance of quick-SOFA (qSOFA) score in predicting severe sepsis and mortality - a prospective study of patients admitted with infection to the emergency department. Scand J Trauma Resusc Emerg Med. 2017;25(1):56.

31. Burnham JP, Kollef MH. qSOFA score: predictive validity in Enterobacteriaceae bloodstream infections. J Crit Care. 2017;43:143-147.

32. Dellinger RP, Carlet JM, Masur H, et al; Surviving Sepsis Campaign Management Guidelines Committee. Surviving Sepsis Campaign guidelines for management of severe sepsis and septic shock. Crit Care Med. 2004;32(3):858-873. Review. Erratum in: Crit Care Med 2004;32(10):2169-2170.

33. Aikawa N, Fujishima S, Endo S, et al. Multicenter prospective study of procalcitonin as an indicator of sepsis. $J$ Infect Chemother. 2005;11(3):152-159.

34. Sugimoto K, Adomi S, Koike H, Esa A. Procalcitonin as an indicator of urosepsis. Res Rep Urol. 2013;5:77-80.

35. van Nieuwkoop C, Bonten TN, van't Wout JW, et al. Procalcitonin reflects bacteremia and bacterial load in urosepsis syndrome: a prospective observational study. Crit Care. 2010;14(6):R206. 
36. Ferrer R, Martin-Loeches I, Phillips G. Empiric antibiotic treatment reduces mortality in severe sepsis and septic shock from the first hour: results from a guideline-based performance improvement program. Crit Care Med. 2014;42(8):1749-1755.

37. Karam G, Chastre J, Wilcox MH, Vincent JL. Antibiotic strategies in the era of multidrug resistance. Crit Care. 2016;20(1):136.

38. Joshi S. Hospital antibiogram: a necessity. Indian J Med Microbiol. 2010;28(4):277-280.

39. Elhanan G, Sarhat M, Raz R. Empiric antibiotic treatment and the misuse of culture results and antibiotic sensitivities in patients with community-acquired bacteraemia due to urinary tract infection. J Infect. 1997;35(3):283-288.

40. Rivers E, Nguyen B, Havstad S, et al; Early Goal-Directed Therapy Collaborative Group. Early goal-directed therapy in the treatment of severe sepsis and septic shock. NEngl JMed. 2001;345(19):1368-1377.

41. Kalra OP, Raizada A. Approach to a patient with urosepsis. J Glob Infect Dis. 2009;1(1):57-63.

42. Park JM, Bloom DA. The pathophysiology of ureteropelvic junction obstruction. Urol Clin North Am. 1998;25:161-169.

43. Vigil HR, Hickling DR. Urinary tract infection in the neurogenic bladder. Transl Androl Urol. 2016;5(1):72-87.

44. Knopf HJ, Graff HJ, Schulze H. Perioperative antibiotic prophylaxis in ureteroscopic stone removal. Eur Urol. 2003;44(1):115-118.

45. Wolf JS Jr, Bennett CJ, Dmochowski RR, et al. Urologic surgery antimicrobial prophylaxis best practice policy panel. Best practice policy statement on urologic surgery antimicrobial prophylaxis. J Urol. 2008;179(4):1379-1390.
46. Eswara JR, Shariftabrizi A, Sacco D. Positive stone culture is associated with a higher rate of sepsis after endourological procedures. Urolithiasis. 2013;41(5):411-414.

47. Chang CL, Su LH, Lu CM, Tai FT, Huang YC, Chang KK. Outbreak of ertapenem-resistant Enterobacter cloacae urinary tract infections due to a contaminated ureteroscope. J Hosp Infect. 2013;85(2):118-124.

48. Carey RI, Gomez CS, Maurici G, Lynne CM, Leveillee RJ, Bird VG. Frequency of ureteroscope damage seen at a tertiary care center. J Urol. 2006;176:607-610.

49. Ofstead CL, Heymann OL, Quick MR, Johnson EA, Eiland JE, Wetzler HP. The effectiveness of sterilization for flexible ureteroscopes: a realworld study. Am J Infect Control. 2017;45(8):888-895.

50. AORN. Association of Perioperative Registered Nurses Guideline for Processing Flexible Endoscopes Sterilization and Disinfection. Denver, CO: AORN; 2016:675-758.

51. Usawachintachit M, Isaacson DS, Taguchi K, et al. A prospective case-control study comparing LithoVue, a single-use, flexible disposable ureteroscope, with flexible, reusable fiber-optic ureteroscopes. $J$ Endourol. 2017;31(5):468-475.

52. Auge BK, Pietrow PK, Lallas CD, Raj GV, Santa-Cruz RW, Preminger GM. Ureteral access sheath provides protection against elevated renal pressures during routine flexible ureteroscopic stone manipulation. $J$ Endourol. 2004;18(1):33-36.

53. Huang J, Zhao Z, AlSmadi JK, et al. Use of the ureteral access sheath during ureteroscopy: a systematic review and meta-analysis. PLoS One. 2018;13(2):e0193600.
Research and Reports in Urology

\section{Publish your work in this journal}

Research and Reports in Urology is an international, peer-reviewed, open access journal publishing original research, reports, editorials, reviews and commentaries on all aspects of adult and pediatric urology in the clinic and laboratory including the following topics: Pathology, pathophysiology of urological disease; Investigation and treatment of

\section{Dovepress}

urological disease; Pharmacology of drugs used for the treatment of urological disease. The manuscript management system is completely online and includes a very quick and fair peer-review system, which is all easy to use. Visit http://www.dovepress.com/testimonials.php to read real quotes from published authors. 